\title{
THE INFLUENCE OF CISPLATIN DOSE UPON SURVIVAL IN CONCURRENT CHEMORADIOTHERAPY OF LOCALLY ADVANCED CERVICAL CARCINOMA WITH WEEKLY CISPLATIN
}

\author{
Igor Sirák, Jiř́ Petera, Zdeněk Zoul
}

Charles University in Prague, Faculty of Medicine and University Hospital Hradec Králové, Czech Republic: Department of Oncology and Radiotherapy

Summary: The objective of this study was to evaluate the influence of cisplatin dose upon 3-year overall and disease-free survival rate of patients with advanced cervical cancer treated with concurrent chemoradiotherapy with weekly cisplatin. Seventy-three patients with stage IIB - IVA cervical carcinoma were treated with pelvic (or pelvic + paraaortic) externalbeam radiotherapy, high-dose rate brachytherapy and concomitant chemotherapy with weekly cisplatin of $40 \mathrm{mg} / \mathrm{m}^{2}$ in the time period form January 2000 to December 2006 at our department. The 3-year overall survival and disease-free suvival rates were evaluated with regard to the number of cisplatin cycles applied during the external radiotherapy. Only twentyeight patients received the intended five doses of chemotherapy. The most frequent cause of chemotherapy delay was the acute hematological toxicity with leukopenia. The 3-year overall survival was $71 \%$ and the 3-year disease-free survival was $61 \%$. Survival analyses didn't prove a statistically significant influence of cisplatin dose upon 3-year survival in cervical carcinoma patients treated by exclusive chemoradiation with weekly cisplatin.

Key words: Cervical carcinoma; Concurrent chemoradiotherapy; Cisplatin

\section{Introduction}

Carcinoma of the uterine cervix is a common malignancy in the Czech Republic. There were 1082 new cases diagnosed and 399 deaths reported due to this malignancy in 2002. Radical radiotherapy is an established treatment for IIB, III and IVA stage at the time of diagnosis. Despite improvements in radiation techniques, in approximately two thirds of the cases progression occurs within the irradiated area if radiotherapy alone is used. Failure of local-regional control usually results in death. Several randomized studies and two meta-analyses have demonstrated that concurrent chemoradiotherapy of locally advanced cervical carcinoma improves overall and progression-free survival and reduces the incidence of local and distant recurrence compared to radiotherapy alone $(4,8)$. Concomitant cytotoxic therapy applied with radiotherapy has the advantage of greater tumor radiosensitivity as well as better control of distant metastases. Cisplatin, as the most active substance synergistic with radiotherapy, has become a standard component of chemotherapy regimen used concurrently with irradiation. However, the dosage and timing have not been accurately standardized as of yet. The purpose of this retrospective study was to evaluate the influence of cisplatin dose upon the 3-year overall survival and 3-year disease-free survival rate of patients with advanced cervical cancer treated with concurrent chemoradiotherapy with weekly cisplatin.

\section{Materials and Methods}

\section{Patients}

Between January 2000 and December 2006, 73 patients with locally advanced cervical carcinoma were treated with concurrent chemoradiotherapy with weekly cisplatin at the Department of Oncology and Radiotherapy, University Hospital Hradec Králové, Czech Republic. The radical treatment consisted of a 5-week course of external-beam radiotherapy to the small pelvis (or small pelvis plus paraaortal lymph-nodes) with concurrent weekly cisplatin of 40 $\mathrm{mg} / \mathrm{m}^{2}$, followed by high-dose rate brachytherapy with simultaneous external parametrial irradiation performed without concomitant chemotherapy.

The inclusion criteria were as follows: untreated and histologically confirmed squamous cell carcinoma or adenocarcinoma of the uterine cervix, FIGO stage IIB - IVA, WHO performance status of $0-2$, age less than 75 years, adequate hematologic, renal and liver function, patient consent to the treatment. All patients who met the inclusion criteria in the referenced time period were enrolled into our study. 
Tab. 1: Patient and tumor characteristics $(n=73)$.

\begin{tabular}{|c|}
\hline Age \\
\hline $\begin{array}{l}\text { - Average: } 47 \text { years ( } 27-73 \text { years) } \\
\text { - Median: } 50 \text { years }\end{array}$ \\
\hline FIGO stage \\
\hline $\begin{array}{l}\text { - IIB: } 57 \text { patients }(78 \%) \\
\text { - IIIB: } 15 \text { patients }(21 \%) \\
\text { - IVA: } 1 \text { patient }(1 \%)\end{array}$ \\
\hline Histology \\
\hline $\begin{array}{l}\text { - Squamous cell carcinoma: } 69 \text { patients }(95 \%) \\
\text { - Adenocarcinoma: } 4 \text { patients }(5 \%)\end{array}$ \\
\hline Tumor differentiation \\
\hline $\begin{array}{l}\text { - Well: } 4 \text { patients }(5 \%) \\
\text { - Moderate: } 41 \text { patients }(56 \%) \\
\text { - Poor: } 28 \text { patients }(39 \%)\end{array}$ \\
\hline
\end{tabular}

Baseline investigations included clinical and gynecological examinations, tumor biopsy performed during the gynecological evaluation under anaesthesia, hematological and biochemical assessments, chest X-rays, bipedal lymphography, computer tomography and ultrasound of pelvis and abdomen. Cystoscopy and rectosigmoideoscopy was performed only in suspicion of rectal or urinary bladder infiltration.

The majority of patients ( $75 \%$ ) were treated throughout hospitalization, $25 \%$ were out-patients. Patient and tumor characteristics are described in Table 1.

\section{Treatment}

The external-beam radiotherapy (ERT) was provided by linear accelerator, using a photon beam of 6-15 MV energy. A four-field (BOX) technique with individually shaped portals was used containing the pelvis in all 73 patients, and para-aortic lymphatic nodes up to the inferior margin of Th12 in 44 patients with initial pelvic lymph-node involvement. Superior limit of the pelvis was at L4/L5, inferior at ischiac tuberosity, ventral at front of the sharebone, dorsal at sacral concavity, lateral limits were $2 \mathrm{~cm}$ externally from bone structures in transversal diameter. The dose delivered to the small pelvis was $25 \times 2$ Gy in five consecutive weeks. The dose to para-aortic lymh nodes was $22 \times 2$ Gy, delivered at the same time. A high-dose rate brachytherapy (HDR BT) came immediately after 25 fractions of ERT, using a remote afterloading device (Gammamed, MDS Nordion, Hahn, Germany). Six fractions of 4 Gy were administered to the A point. The rectal doses and doses to the urinary bladder were calculated according to the International Commission on Radiation Units (ICRU 38) recommendations. The ERT boost of 7 x 2 Gy was delivered to pelvic walls simultaneously with brachytherapy, with shielding of the region irradiated by brachytherapy.

Weekly cisplatin of $40 \mathrm{mg} / \mathrm{m}^{2}$ was administred concurrently with the course of 25 ERT fractions and did not continue during brachytherapy. Standard antiemetic premedication with appropriate hydratation was administered before cisplatin infusion.
Chemotherapy delay criteria included: white blood count $<2.5 \times 10^{9} / \mathrm{L}$, platelet count $<100 \times 10^{9} / \mathrm{L}$, haemoglobin $<100 \mathrm{~g} / \mathrm{L}$, calculated creatinine clearance $<1,0 \mathrm{~mL} / \mathrm{sec}$, grade 3 or 4 gastrointestinal toxicity, and/or patient's refusal.

Radiotherapy delay criteria included: absolute neutrophil count $<1.0 \times 10^{9} / \mathrm{L}$, platelet count $<50 \times 10^{9} / \mathrm{L}$, haemoglobin $<80 \mathrm{~g} / \mathrm{L}$ and/or grade 4 acute gastrointestinal or genitourinary toxicity.

No colony-stimulating growth factors were used for leukopenia prevention. Low hemoglobin levels were corrected by blood transfusions. No erythropoetin was routinely used to keep the adequate hemoglobin level during radiotherapy.

\section{Follow-up and statistics}

The patients were evaluated for objective response, disease-free survival, overall survival, acute and late toxicity. The objective response was evaluated by gynecological examination three months after the treatment. The patients were followed in 3-month intervals by clinical and gynecological examination, hematological, and biochemical tests. Chest X-rays and CT of the pelvis and abdomen were performed every six months. The overall survival (OS) was calculated from the date of diagnosis to the date of last examination or death. The disease-free survival (DFS) was calculated from the date of treatment termination to the date of relapse disclosure, death, or last examination. The OS and DFS rates were calculated using the Kaplan-Meier method. The 3-year overall and disease-free survival rates were evaluated with regard to the number of cisplatin cycles applied, using logrank test. Univariate and multivariate lognormal parametric survival regression analysis was performed to evaluate the prognostic value of chemotherapy dose. The adverse effects of therapy were classified and evaluated according to the RTOG acute radiation morbidity scoring criteria. Acute toxicity was recorded until 90 days from the treatment initiation.

\section{Results}

All 73 patients were accessible for response and toxicity evaluation. Three months after the treatment, the gynecological examination has confirmed a complete response in 69 patients (95\%), residual macroscopic disease was present in 4 patients ( $5 \%)$. Up to the day of the evaluation ( $1^{\text {th }}$ July 2007) the median duration of follow-up was 24 months (range 5-94 months). With a median follow-up duration of 24 months: 17 patients ( $23 \%$ ) have died, 49 patients $(67 \%)$ were alive and disease-free and 7 patients (10\%) were alive with progressive disease. Pelvic relapse was detected in 6 patients, in 2 of them distant metastases were detected at the same time. A combination of para-aortic lymph node recurrence and distant metastases was detected in 1 patient. Distant metastases with central control of the disease were found in 5 patients. The time to pelvic relapse detection had varied between 6 and 24 months after the treatment, 
the time to distant metastases detection had varied between 4 and 24 months after the treatment. Distant seeding occurred predominantly in lungs, liver, peritoneum, axial skeleton and supraclavicular lymphnodes.

Only one patient did not complete the whole schedule of radiotherapy, as brachytherapy had to be replaced with another $5 \times 2$ Gy of ERT due to insufficient tumor regression. In another three patients, the course of ERT had to be delayed: once due to leukopenia alone (after $4^{\text {th }}$ dose of cisplatin, treatment break for 7 days), twice due to protracted leukopenia with the trombocytopenia (in both after $2^{\text {nd }}$ dose of chemotherapy, treatment break once for 14 days and once for 21 days). No radiotherapy dose recovery was performed in these patients. Radiotherapy mean treatment time was 7 weeks.

Only 28 patients (38\%) were able to undergo the intended dose rate ( 5 cycles) of cisplatin. Table 2 shows the detail survey of applied chemotherapy. The most frequent cause of chemotherapy delay was hematological toxicity (in 30 cases): leukopenia alone in 20 patients, combination of

Tab. 2: Cycles of cisplatin applied.

\begin{tabular}{|c|c|c|}
\hline Cycles of cisplatin & Patients $(\mathrm{n}=73)$ & Proportion \\
\hline 5 & 28 & $38 \%$ \\
\hline 4 & 16 & $22 \%$ \\
\hline 3 & 15 & $21 \%$ \\
\hline 2 & 12 & $16 \%$ \\
\hline 1 & 2 & $3 \%$ \\
\hline
\end{tabular}

leukopenia and thrombocytopenia in 8 patients, thrombocytopenia alone in 2 patients. In 7 patients the chemotherapy was stopped because of low creatinine clearance. Gastrointestinal toxicity caused delay in 5 patients, one delay was due to the patient's refusal (fatigue, weakness).

Acute hematological toxicity was observed in 69 patients, mostly of grade 2 or 3 . Two patients experienced a febrile neutropenia (both after $3^{\text {th }}$ dose of cisplatin). Acute gastrointestinal and urogenital toxicity was mostly of grade 1 or 2 . No grade 4 non-hematological acute toxicity was observed. No death developed from the acute toxicity. Table 3 shows the detailed survey of acute treatment toxicity.

The 3-year overall survival was $71 \%$ and the 3-year disease-free survival was $61 \%$. Differences in survival rates of group variables, according to the number of cisplatin cycles applied, are described in Table 4 and Table 5.

Of all these comparisons, only one (OS for 5 vs. < 4 cycles) was significant. This comparison was corrected by multiple Cox survival regression analysis of potential tumor and patient-related factors: lymph-node involvement $(\mathrm{p}=$ $0.22)$, cisplatin dose $(p=0.63)$, age $(p=0.76)$, FIGO stage $(\mathrm{p}=0.83)$, tumor grade $(\mathrm{p}=0.85)$.

Univariate lognormal parametric survival regression showed no significant influence on the number of cisplatin cycles applied upon overall survival $(p=0.69)$, as well as multivariate parametric regression, when prognostic factors of age, tumor grade and histology, FIGO stage and lymph-

Tab. 3: Acute treatment toxicity $(n=73)$.

\begin{tabular}{|c|c|c|c|c|c|}
\hline Acute toxicity & Leucopenia & Thrombocytopenia & Upper GI tract & Lower GI tract & Urinogenital tract \\
\hline Grade 0 & $4(5 \%)$ & $48(66 \%)$ & $43(59 \%)$ & $23(32 \%)$ & $49(67 \%)$ \\
\hline Grade 1 & $13(18 \%)$ & $12(16 \%)$ & $17(23 \%)$ & $39(53 \%)$ & $17(23 \%)$ \\
\hline Grade 2 & $30(41 \%)$ & $9(12 \%)$ & $10(14 \%)$ & & $7(10 \%)$ \\
\hline Grade 3 & $24(33 \%)$ & $4(5 \%)$ & $3(4 \%)$ & $11(15 \%)$ & - \\
\hline Grade 4 & $2(3 \%)$ & - & - & - & - \\
\hline
\end{tabular}

Tab. 4: The 3-year overall survival according to cisplatin dose $(n=73)$.

\begin{tabular}{|c|c|c|c|c|c|}
\hline Cisplatin dose & $\mathrm{n}$ & 3-year overall survival & logrank & Hazard Ratio & $95 \% \mathrm{CI}$ \\
\hline $\begin{array}{c}5 \text { cycles } \\
\leq 4 \text { cycles }\end{array}$ & $\begin{array}{l}28 \\
45\end{array}$ & $\begin{array}{l}79 \% \\
67 \%\end{array}$ & $\mathrm{p}=0.01$ & 0.94 & $0.35-2.52$ \\
\hline $\begin{array}{l}\geq 4 \text { cycles } \\
\leq 3 \text { cycles }\end{array}$ & $\begin{array}{l}44 \\
29 \\
\end{array}$ & $\begin{array}{l}63 \% \\
73 \% \\
\end{array}$ & $\mathrm{p}=0.14$ & 1.20 & $0.46-3.12$ \\
\hline $\begin{array}{l}\geq 3 \text { cycles } \\
\leq 2 \text { cycles }\end{array}$ & $\begin{array}{l}59 \\
14 \\
\end{array}$ & $\begin{array}{l}70 \% \\
75 \% \\
\end{array}$ & $\mathrm{p}=0.35$ & 0.99 & $0.32-3.04$ \\
\hline
\end{tabular}

Tab. 5: The 3-year disease-free survival according to cisplatin dose $(n=69)$.

\begin{tabular}{|c|c|c|c|c|c|}
\hline Cisplatin dose rate & $\mathrm{n}$ & 3-year DFS & logrank & Hazard Ratio & $95 \% \mathrm{CI}$ \\
\hline $\begin{array}{c}5 \text { cycles } \\
\leq 4 \text { cycles }\end{array}$ & $\begin{array}{l}28 \\
41\end{array}$ & $\begin{array}{l}74 \% \\
61 \%\end{array}$ & $\mathrm{p}=0.28$ & 0.77 & $0.30-1.96$ \\
\hline $\begin{array}{l}\geq 4 \text { cycles } \\
\leq 3 \text { cycles }\end{array}$ & $\begin{array}{l}42 \\
27 \\
\end{array}$ & $\begin{array}{l}69 \% \\
54 \% \\
\end{array}$ & $\mathrm{p}=0.66$ & 0.69 & $0.28-1.72$ \\
\hline $\begin{array}{l}\geq 3 \text { cycles } \\
\leq 2 \text { cycles }\end{array}$ & $\begin{array}{l}56 \\
13 \\
\end{array}$ & $\begin{array}{l}66 \% \\
54 \% \\
\end{array}$ & $\mathrm{p}=0.08$ & 1.17 & $0.41-3.36$ \\
\hline
\end{tabular}


node involvement were taken into account $(p=0.54)$ Likewise, lognormal parametric survival regression showed no significant effect of cisplatin dose on disease-free survival: univariate $(p=0.34)$, multivariate $(p=0.34)$.

\section{Discussion}

In the presented retrospective study, we tried to evaluate the influence of cisplatin dose upon the 3-year OS and DFS rates in patients with cervical carcinoma treated by external-beam radiotherapy, HDR brachytherapy, and weekly cisplatin. Our results didn't prove a statistically significant influence of cisplatin dose upon survival. Discrepancy between significative OS and no singnificative DFS in patients treated by five cisplatin cycles is most likely accidental. Definitive conclusions cannot be made because of the small number of patients, which doesn't allow excluding differences among subgroups treated.

The meta-analysis of randomized studies, comparing concurrent chemoradiotherapy in locally advanced cervical cancer to radiotherapy alone, has evidenced significantly higher acute toxicity of cisplatin-based concomitant chemoradiation (4). In 2003, the same authors have published a systematic review of the concurrent treatment toxicity which describes a significant increase, especially in grade 3 and 4 gastrointestinal and hematological toxicity, with a twofold increase in white blood cell toxicity and threefold increase of platelet toxicity (6). This review, as well as several recently published studies, has described leukopenia as the most common form of acute toxicity in concurrent chemoradiotherapy with cisplatin $(10,15,17,19)$. Our study has confirmed these results, as we have noted the acute hematological toxicity in $95 \%$ of patients, predominantly grade 2 and 3. The most frequent limiting factor of concurrent chemotherapy was leukopenia, which delayed the treatment in 28 patients (38\%). Other forms of toxicity delayed the treatment in another $24 \%$ of patients. Although the vast majority of patients were hospitalized during treatment with adequate supportive therapy, the acute toxicity was very important. This may be related to too high dose of ERT used in our protocol at that time. Altogether, only $38 \%$ of patients were able to tolerate the complete intended course of chemotherapy in our study.

With respect to randomized studies, which have proven the benefit of concomitant treatment, we can assume that fulfillment of concomitant chemotherapy should have a positive influence on survival $(9,11,14,16,18,20)$. However, even these important studies have described only $60 \%$ toleration of the whole chemotherapy plan. These results show that radical radiotherapy combined with concurrent cisplatin chemotherapy is accompanied by considerable acute toxicity, and many patients are unable to comply with the treatment schedule owing to reasons related to acute toxicity. Although the administration of the full chemotherapy dose may be difficult, the delivery of planned radiotherapy was generally not compromised in these studies. Leuko- penia, as the most common form of acute treatment toxicity, represents the main limiting factor of concurrent chemotherapy. Well-timed termination of chemotherapy, when severe leukopenia occurs, is necessary for preservation of the whole course of radiotherapy, which should be completed in the shortest possible time $(3,7,12)$. Administration of granulocyte colony-stimulating factors during the treatment may be helpful in maintaining the leukocyte level, but their effect hasn't been fully proven yet. To our best knowledge, no previous study has sufficiently described the influence of cisplatin dose rate upon the survival of patients with radically treated cervical carcinoma.

\section{Conclusions}

In our patient group we didn't find any statistically significant influence of cisplatin dose upon 3-year survival in cervical carcinoma patients treated by exclusive chemoradiation with weekly cisplatin. Prospective randomized trials are needed to solve the questionable role of chemotherapy dose in these patients. If there was any convincing evidence of positive influence of the full course of chemotherapy upon survival, then regular administration of colony stimulating growth factors would be well-founded.

\section{Acknowledgements}

This study was supported by the Research Project 00179906 of the Ministry of Health, Czech Republic.

\section{References}

1. Cox DR. Regression model and life tables. J Royal Stat Soc 1972; series B 34:187-220.

2. Eifel PJ. Chemoradiotherapy in the treatment of cervical cancer. Semin Radiat Oncol 2006;16:177-85.

3. Girinsky T, Rey A, Roche B, et al. Overall treatment time in advanced cervical carcinomas: a critical parameter in treatment outcome. Int J Radiat Oncol Biol Phys 1993;27:1051-6.

4. Green JA, Kirwan JM, Tierney JF, et al. Survival and recurrence after concomitant chemotherapy and radiotherapy for cancer of the uterine cervix: a systematic review and meta-analysis. Lancet 2001;358:781-6.

5. Kaplan FL, Meier P. Non parametric estimation from incomplete observations. Am J Stat Assoc 1958;53:457-481.

6. Kirwan JM, Symonds P, Green JA, Tierney J, Collingwood M, Williams CJ. A systematic review of acute and late toxicity of concomitant chemoradiation for cervical cancer. Radiotherap and Oncol 2003;68:217-26.

7. Lanciano MR, Pajak TF, Martz K, Hanks GE. The influence of treatment time on outcome for squamous cell cancer of the uterine cervix treated with radiation: a Patterns-of-Care study. Int J Radiat Oncol Biol Phys 1993;25:391-7.

8. Lukka H, Hirte H, Fyles A, et al. Concurrent Cisplatin-based Chemotherapy plus Radiotherapy for Cervical Cancer - a Meta-analysis. Clinical Oncology 2002; 14:203-12.

9. Morris M, Eifel PJ, Lu J, et al. Pelvic radiation with concurrent chemotherapy compared with pelvic and para-aortic radiation for high risk cervical cancer. N Engl J Med 1999;340:1137-43.

10. Park TK, Kim SN, Kim SW, Kim GE, Suh CO. Concurrent chemotherapy and radiotherapy in invasive cervical cancer patients with high risk factors. J Korean Med Sci 2000;15:436-41.

11. Pearcey R, Brundage M, Drouin P, et al. Phase III trial comparing radical radiotherapy with and without cisplatin chemotherapy in patients with advanced squamous cell cancer of the cervix. J Clin Oncol 2002;20:966-72.

12. Perez CA, Grigsby PW, Castro Vita H, Lockett MA. Carcinoma of the uterine cervix. I. Impact of prolongation of treatment time and timing of brachytherapy on outcome of radiation therapy. Int J Radiat Oncol Biol Phys 1995;32:1275-88.

13. Peto R, Peto J. Asymptomatically efficient rank invariant prosedures. J Royal Stat Soc 1972;135:185-207. 
14. Rose PG, Bundy BN, Watkins EB, et al. Concurrent cisplatin-based radiotherapy and chemotherapy for locally advanced cervical cancer. N Engl J Med 1999; 340:1144-53.

15. Serkies K, Jassem J. Concurrent weekly cisplatin and radiotherapy in routine management of cervical cancer: a report on patient compliance and acute toxicity. Int J Radiat Oncol Biol Phys 2004;60:814-21.

16. Schilder JM, Stehman FB. Concurrent chemotherapy and radiation therapy in primary cancer of the cervix. Curr Oncol Rep 1999;1:41-6.

17. Strauss HG, Kuhnt T, Laban C, et al. Chemoradiation in cervical cancer with cisplatin and high-dose rate brachytherapy combined with external beam radiotherapy. Strahlenther Onkol 2002;178:378-85.
18. Thomas GM. Concurrent chemotherapy and radiation for locally advanced cervical cancer: the new standard of care. Semin Radiat Oncol 2000;10: 44-50.

19. Toita T, Moromizato H, Ogawa K, et al. Concurrent chemoradiotherapy using high-dose-rate intracavitary brachytherapy for uterine cervical cancer. Gynecol Oncology 2005;96:665-70.

20. Whitney CW, Sause W, Bundy BN, et al. Randomized comparison of fluorouracil plus cisplatin versus hydroxyurea as an adjunct to radiation therapy in stage IIB-IVA carcinoma of the cervix with negative paraaortic lymph-nodes: a Gynecologic Oncology Group and Southwest Oncology Group study. J Clin Oncol 1997; 17:1339-48

Submitted February 2008.

Accepted April 2008

\section{Corresponding author:}

Igor Sirák, M. D., Department of Oncology and Radiotherapy,

University Hospital, Sokolská 581, 500 05, Hradec Králové, Czech Republic, sirak@fnhk.cz 